\title{
Urinary Incontinence, CTCAE 5.0
}

National Cancer Institute

\section{Source}

National Cancer Institute. Urinary Incontinence, CT CAE 5.0. NCI Thesaurus. Code C146786.

A disorder characterized by inability to control the flow of urine from the bladder. 